\title{
Universiteit
}

Leiden

The Netherlands

\section{Electrically detected interferometry of Majorana fermions in a topological insulator}

Akhmerov, A.R.; Nilsson, J.; Beenakker, C.W.J.

\section{Citation}

Akhmerov, A. R., Nilsson, J., \& Beenakker, C. W. J. (2009). Electrically detected interferometry of Majorana fermions in a topological insulator. Physical Review Letters, 102(21), 216404. doi:10.1103/PhysRevLett.102.216404

Version: $\quad$ Not Applicable (or Unknown)

License: $\quad$ Leiden University Non-exclusive license

Downloaded from: https://hdl.handle.net/1887/63547

Note: To cite this publication please use the final published version (if applicable). 


\title{
Ehrenfest-Time-Dependent Excitation Gap in a Chaotic Andreev Billiard
}

\author{
İ. Adagideli and C.W. J. Beenakker \\ Instituut-Lorentz, Universiteit Leiden, P.O. Box 9506, 2300 RA Leiden, The Netherlands
}

(Received 12 February 2002; published 14 November 2002)

\begin{abstract}
A semiclassical theory is developed for the appearance of an excitation gap in a ballistic chaotic cavity connected by a point contact to a superconductor. Diffraction at the point contact is a singular perturbation in the limit $\hbar \rightarrow 0$, which opens up a gap $E_{\text {gap }}$ in the excitation spectrum. The time scale $\hbar / E_{\text {gap }} \propto \alpha^{-1} \ln \hbar$ (with $\alpha$ the Lyapunov exponent) is the Ehrenfest time, the characteristic time scale of quantum chaos.
\end{abstract}

DOI: $10.1103 /$ PhysRevLett.89.237002

PACS numbers: 74.50.+r, 05.45.Mt, 73.63.Kv, 74.80.Fp

The density of states in a normal metal is suppressed near the Fermi energy when it is brought into contact with a superconductor. The history of this proximity effect goes back to the 1960s [1]. It was understood early on [2] that the energy range of the suppression is the inverse of the typical life time $\tau_{\mathrm{c}}$ of an electron or hole quasiparticle in the normal metal. This lifetime is finite (even at zero temperature) because an electron is converted into a hole by Andreev reflection at the interface with the superconductor [3]. The energy scale $E_{\mathrm{c}}=\hbar / \tau_{\mathrm{c}}$, known as the Thouless energy, is the product of the mean level spacing $\delta$ in the normal metal and the dimensionless conductance of the contact to the superconductor. (For example, $E_{\mathrm{c}}=N \delta$ for coupling via an $N$-channel ballistic point contact.) The appearance of an excitation gap of the order of the Thouless energy is the essence of the traditional proximity effect.

Some years ago it was realized [4-9] that the proximity effect is essentially different if the normal metal becomes so small and clean that scattering by impurities can be neglected. This applies to a quantum dot in a twodimensional electron gas [10], and because of the resemblance to a billiard (cf. Fig. 1) one speaks of an "Andreev billiard" [11,12]. Depending on the shape of the billiard, the classical dynamics varies between integrable and chaotic. No excitation gap is induced by the proximity effect in an integrable billiard $[4,8]$. An excitation gap does appear in a chaotic billiard $[4,6]$, but its magnitude is given by the Thouless energy only if the chaos sets in sufficiently rapidly $[5,9]$.

The characteristic time scale of quantum chaos is the Ehrenfest time $\tau_{\mathrm{E}}=\alpha^{-1} \ln \left(L / \lambda_{\mathrm{F}}\right)$, defined in terms of the Lyapunov exponent $\alpha$ (being the rate at which nearby trajectories diverge exponentially in time) and the relative magnitude of the Fermi wavelength $\lambda_{\mathrm{F}}=2 \pi / k_{\mathrm{F}}$ and a typical dimension $L$ of the billiard [13]. Chaotic dynamics requires $\alpha^{-1} \ll \tau_{\mathrm{c}}$, but $\tau_{\mathrm{E}}$ could be either smaller or larger than $\tau_{\mathrm{c}}$. In the regime $\tau_{\mathrm{E}} \ll \tau_{\mathrm{c}}$ the excitation gap is set as usual by the Thouless energy. Established techniques (random-matrix theory, nonlinear $\sigma$ model) provide a complete description of this regime [4,14-16]. The opposite regime $\tau_{\mathrm{E}} \gg \tau_{\mathrm{c}}$ has no analog in the con- ventional proximity effect. Random-matrix theory is helpless and this regime has also shown a frustrating resilience to solution by means of the ballistic $\sigma$ model [9]. In particular, no mechanism has yet been demonstrated to produce the hard gap at $\hbar / \tau_{\mathrm{E}}$ conjectured by Lodder and Nazarov [5].

Here we report an attack on this problem by an alternative approach, starting from the semiclassical Andreev approximation to the Bogoliubov-De Gennes (BdG) equation [3]. The limit $\tau_{\mathrm{E}} \rightarrow \infty$ yields the BohrSommerfeld approximation to the density of states [4-6],

$$
\rho_{\mathrm{BS}}(E)=\frac{2}{\delta} \frac{\left(E_{\mathrm{c}} / 4 E\right)^{2} \cosh \left(E_{\mathrm{c}} / 4 E\right)}{\sinh ^{2}\left(E_{\mathrm{c}} / 4 E\right)},
$$

which is gapless (cf. Fig. 1). We have found that diffraction

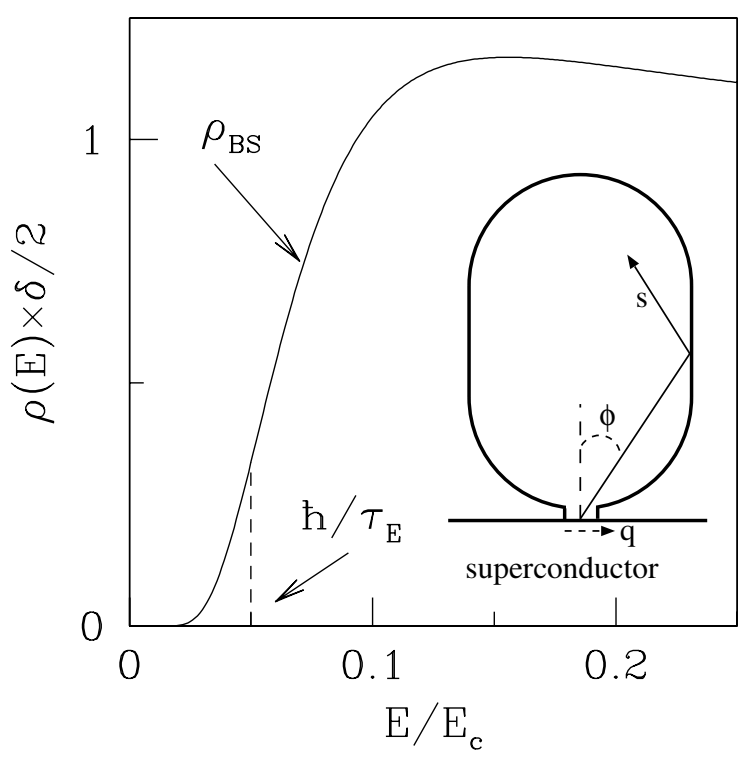

FIG. 1. Solid curve: Density of states $\rho_{\mathrm{BS}}$ of a chaotic Andreev billiard (inset), which is gapless according to the semiclassical Bohr-Sommerfeld approximation (1). The dashed line indicates schematically the phenomenon that we seek to describe in this paper: The opening of a gap at the inverse Ehrenfest time as a result of diffraction at the contact with the superconductor. 
at the contact with the superconductor is a singular perturbation to $\rho_{\mathrm{BS}}$ that opens up a gap at the inverse Ehrenfest time, and provides an intuitively appealing mechanism for the gap phenomenon.

We recall the basic equations. The electron and hole components $u(\mathbf{r})$ and $v(\mathbf{r})$ of the spinor wave function satisfy the BdG equation

$$
\left(\begin{array}{cc}
H & \Delta \\
\Delta^{*} & -H
\end{array}\right)\left(\begin{array}{l}
u \\
v
\end{array}\right)=E\left(\begin{array}{l}
u \\
v
\end{array}\right)
$$

which contains the single-particle Hamiltonian $H=$ $-\nabla^{2}+V(\mathbf{r})-E_{\mathrm{F}}$ (with confining potential $V$ ) and the pair potential $\Delta(\mathbf{r})$ (vanishing in the normal metal and equal to $\Delta_{0}$ in the superconductor). The energy $E$ is measured relative to the Fermi energy $E_{\mathrm{F}}=k_{\mathrm{F}}^{2}$, in units such that $\hbar^{2} / 2 m \equiv 1$. (In these units the mean level spacing $\delta$ is related to the area $\mathcal{A}$ of the billiard by $\delta=$ $4 \pi / \mathcal{A}$.) We assume that the motion inside the billiard is ballistic $(V=0)$ and that the interface with the superconductor is a ballistic point contact of width $W \gg \lambda_{\mathrm{F}}$ (so that the number of channels $N=2 W / \lambda_{\mathrm{F}} \gg 1$ and the Thouless energy $E_{\mathrm{c}}=N \delta \gg \delta$ ). We work in the regime $\Delta_{0} \gg \hbar v_{\mathrm{F}} / W$ (which also implies $\Delta_{0} \gg E_{\mathrm{c}}$ ), to ensure that the excitation spectrum is independent of the properties of the superconductor.

For a semiclassical description one substitutes $(u, v)=$ $(\bar{u}, \bar{v}) A e^{i S}$, with $\hbar S$ the action along a classical trajectory at the Fermi energy. The wave amplitude $A$ is related to the classical action by the continuity equation $\nabla$. $\left(A^{2} \nabla S\right)=0$, while $S$ itself satisfies the Hamilton-Jacobi equation $|\nabla S|^{2}=E_{\mathrm{F}}-V$ (so that $\hbar \nabla S$ is the momentum along the trajectory). The BdG equation takes the form

$$
\left(\begin{array}{cc}
-2 i k_{\mathrm{F}} \partial_{s}+\delta H & \Delta \\
\Delta^{*} & 2 i k_{\mathrm{F}} \partial_{s}-\delta H
\end{array}\right)\left(\begin{array}{c}
\bar{u} \\
\bar{v}
\end{array}\right)=E\left(\begin{array}{c}
\bar{u} \\
\bar{v}
\end{array}\right),
$$

with $\delta H \bar{u}=-A^{-1} \nabla^{2}(A \bar{u})$. The derivative $\partial_{s}=$ $k_{\mathrm{F}}^{-1}(\nabla S) \cdot \nabla$ is taken along the classical trajectory. The Andreev approximation consists in neglecting the term $\delta H$ containing second derivatives of the slowly varying functions $A, \bar{u}, \bar{v}$.

We consider a classical trajectory that starts as an electron at a point $q \in(0, W)$ along the interface with the superconductor, making an angle $\phi \in(-\pi / 2, \pi / 2)$ with the normal (cf. Fig. 1). The product $b=q \cos \phi$ is the "impact parameter." The trajectory returns to the interface after a path length $\ell$, and then it is retraced in the opposite direction as a hole. The coordinate $s \in(0, \ell)$ runs along one repetition of this trajectory. We count trajectories with measure $d q d \sin \phi=d b d \phi$, corresponding to a uniform measure in phase space. Equivalently, we can sum over scattering channels $n=$ $1,2, \ldots N$, related to $\phi$ by $n \approx N|\sin \phi|$.

If we ignore the term $\delta H$ in Eq. (3) we recover the Bohr-Sommerfeld density of states [4-6]. Indeed, without $\delta H$ the solution of the eigenvalue problem is

$$
\left(\begin{array}{c}
\bar{u}_{m} \\
\overline{\boldsymbol{v}}_{m}
\end{array}\right)=\frac{1}{\sqrt{2 \ell}}\left(\begin{array}{c}
e^{i m \pi s / 2 \ell} \\
i e^{-i m \pi s / 2 \ell}
\end{array}\right), \quad E_{m}=m \pi k_{\mathrm{F}} / \ell,
$$

with $m= \pm 1, \pm 3, \pm 5 \ldots$ running over positive and negative odd integers. The path length $\ell$ in a chaotic billiard varies in a quasirandom way upon varying the initial conditions $q$ and $\phi$, with an exponential distribution $P(\ell)=\bar{\ell}^{-1} \exp (-\ell / \bar{\ell})$. (The mean path length is $\bar{\ell}=$ $4 \pi k_{\mathrm{F}} / E_{\mathrm{c}}$ [17].) The density of states

$$
\rho(E)=N \int_{0}^{\infty} d \ell P(\ell) \sum_{m=1,3,5}^{\infty} \delta\left[E-E_{m}(\ell)\right]
$$

then evaluates to the $\rho_{\mathrm{BS}}$ of Eq. (1).

The key assumption that will enable us to go beyond the Andreev and Bohr-Sommerfeld approximations is to assume that the amplitude $A$ varies more slowly in space than the spinor components $\bar{u}$ and $\bar{v}$, so that we can approximate $\delta H$ by $-\nabla^{2}$ (neglecting derivatives of $A$ ). The characteristic length scale $L_{A}$ for the spatial dependence of $A$ is set by the smoothness of the confining potential $V$, while the characteristic length scale for $\bar{u}, \bar{v}$ is the contact width $W$. By assuming $L_{A} \gg W$ we consider the case that diffraction occurs predominantly at the interface with the superconductor, rather than inside the billiard. Since $A$ depends on the shape of the billiard, this is the regime in which we can hope to obtain a geometry-independent "universal" result.

To investigate the effect of $\delta H$ we restrict the dimensionality of the Hilbert space in two ways: First, we neglect any mixing of the $N$ scattering channels. (This is known to be a good approximation of the diffraction that occurs when a narrow constriction opens abruptly into a wide region [18]; it does not require smooth corners in the contact.) Second, since we are interested in excitation energies $E \ll E_{\mathrm{c}}$, we include only the two lowest eigenstates $m= \pm 1$ of the zeroth-order solution (4). [The contributions from higher levels are smaller by a factor $\exp \left(-E_{\mathrm{c}} / 2 E\right)$.] We need to include both $E_{1}$ and $E_{-1}$, although the excitation spectrum contains only positive eigenvalues, because of the (virtual) transitions between these two levels induced by $\delta H$. With these restrictions we have for each scattering channel a one-dimensional eigenvalue problem. The effective Hamiltonian $\mathcal{H}_{\text {eff }}$ is a $2 \times 2$ matrix differential operator acting on functions of $b$.

We write $\mathcal{H}_{\text {eff }}=\mathcal{H}_{0}+\mathcal{H}_{1}$, where $\mathcal{H}_{0}$ corresponds to the Andreev approximation and $\mathcal{H}_{1}$ contains the diffractive effects. The zeroth-order term is diagonal,

$$
\mathcal{H}_{0}=\left(\begin{array}{cc}
\pi k_{\mathrm{F}} / \ell(b) & 0 \\
0 & -\pi k_{\mathrm{F}} / \ell(b)
\end{array}\right) .
$$

The relation between $\ell$ and $b$ is determined by the differential equation $d \ell / d b=g(b) \exp (\kappa \ell)$, which expresses the exponential divergence of nearby trajectories (in terms of a Lyapunov exponent $\kappa=\alpha / v_{\mathrm{F}}$ given as inverse length rather than inverse time). The preexponential $g(b)$ 
is of order unity, changing sign at extrema of $\ell(b)$. Upon integration one obtains

$$
\kappa \ell(b)=-\ln |\kappa b|+\mathcal{O}(1), \quad|\kappa b| \ll 1,
$$

where we have shifted the origin of $b$ such that $b=0$ corresponds to a local maximum $\ell_{\max } \gg \bar{\ell}$ of $\ell(b)$. [The logarithmic singularity is cut off at $|\kappa b| \lesssim$ $\exp \left(-\kappa \ell_{\max }\right)$.] There is an exponentially large number $\mathcal{N}(\ell) \propto \exp (\kappa \ell-\ell / \bar{\ell})$ of peaks around which Eq. (7) applies.

To obtain the diffractive correction $\mathcal{H}_{1}$, in the regime that $\delta H=-\nabla^{2}$, we express the Laplacian in the local displacements $d s$ and $d b$ for fixed $\phi$. The functional form of the transformation is

$$
\mathbf{x}=\mathbf{x}_{\phi}(s, b),
$$

where $\mathbf{x}_{\phi}(s, b)$ is the classical trajectory specified by the initial (i.e., $s=0$ ) direction $\phi$ and impact parameter $b$. The resulting partial differential operators are as follows: (i) $\partial_{s}^{2}$, which has a prefactor of order 1 ; (ii) $\partial_{s} \partial_{b}$, which has a prefactor proportional to $\left(\partial_{s} \mathbf{x}_{\phi} \cdot \partial_{b} \mathbf{x}_{\phi}\right)$; and (iii) $\partial_{b}^{2}$ which has a prefactor proportional to $\left|\partial_{b} \mathbf{x}_{\phi}\right|^{-2} \simeq e^{-2 \kappa s}$. The first term $\partial_{s}^{2}$ is a small correction to the zeroth-order density of states. The second term has a prefactor that is rapidly fluctuating with $s$ and has zero average, thus will be subdominant. The third term, in contrast, is a singular perturbation because it associates a kinetic energy with the variable $b$. The resulting zero-point motion implies a nonzero ground state energy, and hence it is responsible for the opening of an excitation gap. Projecting $\mathcal{H}_{1}$ (with $\partial H=-e^{-2 \kappa s} \partial_{b}^{2}$ ) onto the space spanned by the two lowest eigenfunctions $n= \pm 1$ of Eq. (4), and retaining only the leading order terms in $1 / \kappa \ell$, we find

$$
\mathcal{H}_{1}=\left(\begin{array}{cc}
0 & i \\
-i & 0
\end{array}\right) \frac{\pi}{4} \frac{d}{d b}[\kappa \ell(b)]^{-2} \frac{d}{d b}+\mathcal{O}(\kappa \ell)^{-3} .
$$

The effective Hamiltonian can be brought into a more familiar form by the unitary transformation $\mathcal{H}_{\text {eff }} \rightarrow$ $e^{-i \sigma_{1} \pi / 4} \mathcal{H}_{\text {eff }} e^{i \sigma_{1} \pi / 4}$ (with $\sigma_{i}$ a Pauli matrix), followed by the change of variable $x=\kappa b-\kappa b \ln |\kappa b|$ (in the range $|x|<1$ ). We work again to leading order in $1 / \kappa \ell$, and find

$$
\mathcal{H}_{\text {eff }}=\pi k_{\mathrm{F}} \kappa\left(\begin{array}{cc}
-\epsilon \partial_{x}^{2} & -i / \ln |x| \\
i / \ln |x| & \epsilon \partial_{x}^{2}
\end{array}\right), \quad \epsilon \equiv \frac{\kappa}{4 k_{\mathrm{F}}} .
$$

This effective Hamiltonian has the same form as the BdG Hamiltonian (2), for a fictitious one-dimensional system having $V=E_{\mathrm{F}}$ and having a pair potential $\Delta(x)$ that vanishes logarithmically $\propto 1 / \ln |x|$ at the origin (cf. Fig. 2). The kinetic energy $\epsilon \partial_{x}^{2}$ gives a finite excitation gap, even though $\epsilon \ll 1$. Let us now compute this gap.

Since $e^{i \sigma_{2} \pi / 4} \mathcal{H}_{\text {eff }}^{2} e^{-i \sigma_{2} \pi / 4}$ is a diagonal matrix, the spectrum of $\mathcal{H}_{\text {eff }}$ is given by the scalar eigenvalue problem

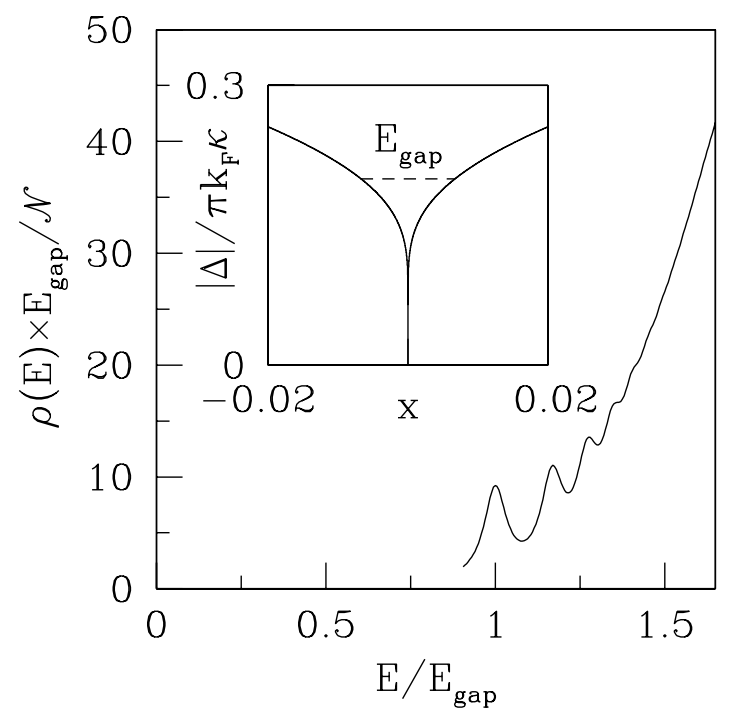

FIG. 2. Low-energy density of states $\rho(E)$ of the effective Hamiltonian (10), related to $\rho(\omega)$ of the biharmonic Eq. (14) by Eq. (18). The plot is for $|\ln \epsilon|=10$ and has been smoothed with a Lorentzian. The inset shows the logarithmic pair potential appearing in $\mathcal{H}_{\text {eff }}$, the ground state of which is the excitation gap (dashed line).

$$
\left|\epsilon \frac{d^{2}}{d x^{2}}+\frac{i}{\ln |x|}\right|^{2} \Psi(x)=\left(\frac{E}{\pi k_{\mathrm{F}} \kappa}\right)^{2} \Psi(x) .
$$

The ground state energy is the excitation gap $E_{\text {gap }}$. To generate an asymptotic expansion of $E_{\text {gap }}$ for small $\epsilon$, we first multiply both sides of Eq. (11) by a factor $Z^{2}$ and then substitute $x=X \sqrt{\epsilon} \bar{Z}$. This results in

$$
\begin{gathered}
\left|\frac{d^{2}}{d X^{2}}+i U\right|^{2} \Psi=\left(\frac{Z E}{\pi k_{\mathrm{F}} \kappa}\right)^{2} \Psi, \\
U(X)=\frac{2 Z}{\ln \epsilon Z}\left[1-\frac{2 \ln |X|}{\ln \epsilon Z}+\mathcal{O}(\ln \epsilon Z)^{-2}\right] .
\end{gathered}
$$

We now choose $Z$ such that $Z^{2}=-\ln ^{3} \epsilon Z$ and obtain the biharmonic equation

$$
\begin{gathered}
\left(d^{4} / d X^{4}+16 \ln |X|\right) \Psi=\omega \Psi, \\
\omega=\left(Z E / \pi k_{\mathrm{F}} \kappa\right)^{2}-4 Z^{2 / 3}+\mathcal{O}\left(Z^{-1 / 3}\right) .
\end{gathered}
$$

The ground state of Eq. (14) is at $\omega_{0}=14.5$. Substituting in Eq. (15), and using $Z^{2 / 3}=|\ln \epsilon|-$ $\frac{3}{2} \ln |\ln \epsilon|+\mathcal{O}(1 / \ln \epsilon)$, we arrive at

$$
E_{\text {gap }}=\frac{2 \pi k_{\mathrm{F}} \kappa}{|\ln \epsilon|}\left[1+\frac{3 \ln |\ln \epsilon|}{2|\ln \epsilon|}+\frac{\omega_{0}}{8|\ln \epsilon|}+\mathcal{O}(\ln \epsilon)^{-3 / 2}\right] .
$$

Only the leading order term is significant in view of the approximations made in Eq. (10). Restoring the original variables we have 


$$
E_{\text {gap }}=\frac{\pi \hbar \alpha}{\ln \left(v_{\mathrm{F}} / \alpha \lambda_{\mathrm{F}}\right)} .
$$

The Ehrenfest time $\tau_{\mathrm{E}}=\alpha^{-1} \ln \left(L / \lambda_{\mathrm{F}}\right)$ contains the classical length $L=v_{\mathrm{F}} / \alpha$, which is of the order of the linear dimension of the billiard.

The density of states $\rho(\omega)$ of the biharmonic Eq. (14) can be calculated numerically [19]. The density of states $\rho(E)$ near the gap is related to $\rho(\omega)$ by

$$
\rho(E)=\frac{8 \mathcal{N}|\ln \epsilon|}{E_{\text {gap }}} \rho\left[\omega=\omega_{0}+8|\ln \epsilon|\left(E / E_{\text {gap }}-1\right)\right],
$$

and is plotted in Fig. 2 for $|\ln \epsilon|=10$. The factor $\mathcal{N} \propto$ $\exp \left(\pi k_{\mathrm{F}} \kappa / E_{\text {gap }}-E_{\mathrm{c}} / E_{\text {gap }}\right)$ counts the number of peaks in $\ell(b)$ around which $\mathcal{H}_{\text {eff }}$ applies. The Bohr-Sommerfeld approximation (1) corresponds to the large- $\omega$ asymptote $\rho(\omega)=\frac{1}{16} \exp (\omega / 16)$. Since $\omega-\omega_{0} \gg 1$ implies $E / E_{\text {gap }}-1 \gg 1 /|\ln \epsilon|$, the width $\Delta E \simeq E_{\text {gap }} /|\ln \epsilon|$ of the energy range above the gap in which the BohrSommerfeld approximation breaks down is small compared to the gap itself.

Because $\mathcal{H}_{\text {eff }}$ has only a few levels in the range $\Delta E$, the density of states $\rho(E)$ oscillates strongly in this range. The levels are highly degenerate (by a factor $\mathcal{N}$ ) in our approximation. Tunneling between the levels will remove the degeneracy and smooth the oscillations. (A small amount of smoothing has been inserted by hand in Fig. 2.) These density of states oscillations with a period set by the Ehrenfest time are reminiscent of those found by Aleiner and Larkin in the energy level correlation function of a normal metal [13].

In conclusion, we have analyzed a mechanism for the "gap phenomenon" in the proximity effect of chaotic systems. Diffraction at the contact with the superconductor is described by an effective Hamiltonian $\mathcal{H}_{\text {eff }}$ that contains (i) a kinetic energy which vanishes in the classical limit and (ii) a pair potential with a logarithmic profile. The resulting excitation gap $E_{\text {gap }}$ (being the ground state energy of $\mathcal{H}_{\text {eff }}$ ) vanishes logarithmically as the ratio of the Fermi wavelength and a classical length scale (set by the Lyapunov exponent) goes to zero. The time scale $\hbar / E_{\text {gap }}$ is the Ehrenfest time, providing a manifestation of quantum chaos in the superconducting proximity effect.
This work was supported by the Dutch Science Foundation NWO/FOM. We thank A. N. Morozov and P. G. Silvestrov for helpful discussions.

[1] P. G. de Gennes, Rev. Mod. Phys. 36, 225 (1964).

[2] W. L. McMillan, Phys. Rev. 175, 537 (1968).

[3] A. F. Andreev, Sov. Phys. JETP 19, 1228 (1964).

[4] J. A. Melsen, P.W. Brouwer, K. M. Frahm, and C.W. J. Beenakker, Europhys. Lett. 35, 7 (1996); Phys. Scripta T69, 223 (1997).

[5] A. Lodder and Yu. V. Nazarov, Phys. Rev. B 59, 5783 (1998).

[6] H. Schomerus and C.W. J. Beenakker, Phys. Rev. Lett. 82, 2951 (1999).

[7] S. Pilgram, W. Belzig, and C. Bruder, Phys. Rev. B 62, 12462 (2000).

[8] W. Ihra, M. Leadbeater, J. L. Vega, and K. Richter, Europhys. J. B 21, 425 (2001); W. Ihra and K. Richter, Physica (Amsterdam) 9E, 362 (2001).

[9] D. Taras-Semchuk and A. Altland, Phys. Rev. B 64, 014512 (2001).

[10] B. J. van Wees and H. Takayanagi, in Mesoscopic Electron Transport, edited by L. L. Sohn, L.P. Kouwenhoven, and G. Schön, NATO ASI Series E345 (Kluwer, Dordrecht, 1997).

[11] I. Kosztin, D. L. Maslov, and P. M. Goldbart, Phys. Rev. Lett. 75, 1735 (1995); İ. Adagideli and P. M. Goldbart, Phys. Rev. B 65, 201306 (2002).

[12] J. Wiersig, Phys. Rev. E 65, 036221 (2002).

[13] I. L. Aleiner and A. I. Larkin, Phys. Rev. B 54, 14423 (1996); Phys. Rev. E 55, R1243 (1997).

[14] M. G. Vavilov, P.W. Brouwer, V. Ambegaokar, and C.W. J. Beenakker, Phys. Rev. Lett. 86, 874 (2001).

[15] P. M. Ostrovsky, M. A. Skvortsov, and M.V. Feigelman, Phys. Rev. Lett. 87, 027002 (2001).

[16] A. Lamacraft and B. D. Simons, Phys. Rev. B 64, 014514 (2001).

[17] W. Bauer and G. F. Bertsch, Phys. Rev. Lett. 65, 2213 (1990).

[18] A. Szafer and A. D. Stone, Phys. Rev. Lett. 62, 300 (1989).

[19] L. Greenberg and M. Marletta, ACM Trans. Math. Softw. 23, 453 (1997). The code is available at http://www. netlib.org/toms/775 